\title{
LAS CUALIDADES DE LOS OBJETOS INFLUYEN EN EL TIEMPO DE REACCIÓN DE LAS PERSONAS CON ENFERMEdAd de PARKINSON*
}

\section{THE QUALITIES OF THE OBJECTS INFLUENCE THE REACTION TIME IN PEOPLE WITH PARKINSON'S DISEASE}

\author{
María de los Angeles Bacigalupe ${ }^{* *}$, María Paula Tujague*** \\ LUIS EDUARDO CASTRO***
}

\begin{abstract}
${ }^{*}$ Trabajo financiado por el Consejo Nacional de Investigaciones Científicas y Técnicas (CONICET). **Profesora y Licenciada en Ciencias de la Educación y Doctora en Ciencias de la Salud. Codirectora del Programa Estable Taller de Parkinson de la Facultad de Ciencias Médicas de la Universidad Nacional de La Plata (UNLP). Miembro de la Carrera del Investigador Científico del Consejo Nacional de Investigaciones Científicas y Técnicas (CONICET).

E-Mail: mariabacigalupe@conicet.gov.ar, mangelesbacigalupe@gmail.com

***Doctora en Ciencias Naturales y Licenciada en Biología. Investigadora Asociada del Instituto de Biología Subtropical (IBS) - Consejo Nacional de Investigaciones Científicas y Técnicas (CONICET), Universidad Nacional de Misiones (UNAM) y Centro de Investigaciones del Bosque Atlántico (CelBA), Puerto Iguazú (Prov. de Misiones). ${ }^{* * * * D o c t o r}$ en Ciencias Naturales, Profesor de Estadística y Licenciado en Biología. Profesor Titular de Geofísica, Profesor Adjunto de Estadística e Investigador de la Facultad de Ciencias Naturales y del Museo de la Universidad Nacional de La Plata (UNLP). La Plata.

Los autores agradecen a los pacientes con Enfermedad de Parkinson su participación en el estudio, como así también la dirección académica al Dr. H. Lahitte, Profesor Titular y Jefe de la División Etnografía del Museo de La Plata (FCNyM, UNLP), la asistencia del Neurólogo Dr. J.L. Dillon, ex-Jefe de Servicio de Neurología del Hospital Interzonal Dr. A. Korn de Melchor Romero de La Plata y creador del Programa Taller de Parkinson (FCM, UNLP) y de la Psiquiatra Dra. S. Pujol, Profesora Adjunta y Directora del Programa Taller de Parkinson (FCM, UNLP). También agradecen las valiosas sugerencias del Dr. C. Garay, Profesor Adjunto de la Facultad de Psicología (UNLP) y de la Ing. Romina Istvan, Jefe de Trabajos Prácticos de la Universidad Tecnológica Nacional (UTN).

División Etnografía del Museo de La Plata (Facultad de Ciencias Naturales y Museo de la Universidad Nacional de La Plata) Programa Estable Taller de Parkinson (Facultad de Ciencias Médicas de la Universidad Nacional de La Plata) Provincia de Buenos Aires, República Argentina.
\end{abstract}

\section{RESUMEN}

La Enfermedad de Parkinson (EP) constituye un trastorno motor que presenta síntomas motores y no motores, incluyendo rigidez, bradiquinesia, alteración de reflejos posturales, temblor de reposo, trastornos del sueño, de la deglución y de la expresión oral, facial y corporal, con una presentación y severidad variable. En la EP el mo- vimiento en sí está preservado y lo que resulta afectada es la modulación del mismo. Sin embargo, esta enfermedad presenta una característica particular que es la kinesia paradojal, una cualidad del sistema motor por la cual las personas pueden moverse de forma inesperada, considerando las limitaciones atribuidas a la enfermedad. Si bien esta característica paradojal es una propiedad del sistema motor per se, aparece de forma 
más notoria en los trastornos del movimiento que en el comportamiento motor sano y se asocia a los estímulos ambientales. Se presenta un estudio controlado de generalización limitada en el cual se utilizó una aplicación informática con el propósito de evaluar el efecto de las affordances (un recurso ambiental que provee al individuo una oportunidad de comportarse) sobre el tiempo de reacción en un grupo de personas con EP y un grupo control. Los resultados mostraron que los sujetos con EP presentaron un mayor tiempo de reacción que los controles, pero también mostraron aprendizaje a lo largo de la tarea y una mayor sensibilidad al efecto sensorial de compatibilidad. Los resultados obtenidos permiten formular hipótesis sobre los efectos paradójicos que el ambiente puede ejercer al combinarse con las potencialidades de personas con EP.

Palabras clave: Kinesia paradojal; Affordances; Enfermedad de Parkinson; Ambiente; Aprendizaje.

\section{ABSTRACT}

Parkinson's disease (PD) is a movement disorder with motor and non-motor symptoms, including rigidity, bradykinesia, postural instability, resting tremor, sleep disorders, difficulty swallowing, vegetative symptoms, speech problems and body and facial expression disturbances. Symptoms and disease severity are variable across the patients. Besides, the motion is preserved but the problems of modulation hinder its expression. However, the disease has a particular characteristic called paradoxical kinesia, a quality of the motor system by which people is able to unexpectedly move, contrarily to what is commonly expected for people suffering the inabilities of the disease. Although this paradoxical property is a property of the motor system per se, it is more apparent in movement disorders than in healthy motor behavior and is associated with the environmental stimuli. Several scientific reports have proposed that instead of having movement and perception systems separately, they are integrated in a called action-perception system. Besides, researchers have shown that the environmental scenarios have action properties in relation to this actor actionperception system. For example, a ball in the air has the property of catchability in relation to the perception of the individual, which, in turn, is related to his / her movement. This is called affordance, an environmental resource which provides the individual an opportunity to behave. An affordance represents a relationship between the individual action-perception system with the environmental qualities or learning contexts. We develop a controlled study with limited generalization using a computational tool to evaluate the effect of affordances on reaction time in a group of people with PD and a control group. In each trial an arrow indicating a given direction (right or left) was superposed to a previously displayed image of a frying pan with a handle directed to the right or the left side of the screen. Participants were told to press a key of the side of the keyboard that matches with the arrow direction. It was supposed that the direction of the handle would play the role of an affordance to the perception of the individual; therefore participants could find a conflict between directions of the arrow and the handle. Trial conditions could be two: (a) Compatibility: the directions of the arrow and the handle matched and (b) Incompatibility: those directions did not match. Learning was defined by the decrease of reaction time along the trials. We used a Generalized Linear Model (GLM) to explore the relationship between variables and Generalized Linear Mixed Model with repeated measures (MGLM) to model the incidence of successive trials on reaction time. The complete sample included 1050 trials from 4 PD patients and 4 control subjects. Our results showed that (a) reaction times increased with the presence of PD and age of subjects, (b) there was a high incidence of repetition of trials on reaction times of PD and control subjects, and (c) the compatibility between the stimulus and the image affected reaction time on PD patients exclusively. These results indicate that PD patients were able to learn along trials and this learning was favored by an appropriated sensorial arrangement of the stimuli or compatibility effect. Our results let us hypothesize about the paradoxical effects that the environment can exert on people with PD by its combination with their potentialities. Compatibility trials in our study represent appropriate arrangement of stimuli that have been proven to be necessary for the occurrence of paradoxical kinesia. Given that possibility, we can generate learn- 
ing contexts to help people with PD to develop these behavioral phenomena in order to improve their movement, their relation with their environment and also their quality of life.

Key words: Paradoxical kinesia; Affordances; Parkinson's disease; Environment; Learning.

\section{INTRODUCCIÓN}

LA KINESIA PARADOJAL EN LA ENFERMEDAD DE PARKINSON

La Enfermedad de Parkinson (EP) constituye una condición idiopática (de causa desconocida pero que probablemente incluye variables ecogenéticas) clasificada, desde el punto de vista médico, como trastorno del movimiento. Según su prevalencia, se ubica en segundo lugar dentro de las enfermedades neurodegenerativas, aumentando a medida que aumenta la edad del grupo etario considerado (Pringsheim, Jette, Frolkis \& Steeves, 2014).

La medida de prevalencia es variable en las distintas regiones geográficas e informes científicos. A modo de ejemplo, se pueden mencionar los siguientes datos (cifras siempre sobre 100.000 habitantes): (a) en Ciudad Autónoma de Buenos Aires, 219 en la población general y 394 en la población mayor de 40 años (Bauso et al., 2012), (b) en Sudamérica, 228 en la población de 50 a 59 años, 637 en el grupo de 60-69 años, 2.180 en el grupo de 70-79 años y 6.095 en el grupo de 80 y más años (Pringsheim et al., 2014).

La EP se caracteriza por sus llamados síntomas motores (bradiquinesia, alteración de reflejos posturales, temblor de reposo, rigidez), aunque también se consideran parte del cuadro clínico, síntomas no motores (emocionales, cognitivos, vegetativos). Las personas con EP tienen movimiento pero la modulación del mismo se ve afectada como consecuencia del trastorno anátomo-patológico. Los síntomas motores y no motores provocan alteraciones funcionales en la vida diaria de estos pacientes, afectando su comunicación con el medio (Holtgraves \& Cadle, 2016). Con respecto a la cuestión de la comunicación y la acción humanas, es un problema que involucra a las ciencias de la salud y al comportamiento: tanto el trastorno de la movilidad per se como la presencia de signos no motores y sus consecuencias tienen una enorme influencia sobre la calidad de vida del sujeto al alterar sus relaciones con el entorno.

Desde una perspectiva enfocada sobre las relaciones y no sobre el déficit individual, la EP presenta la oportunidad única de analizar una característica del sistema motor, denominada kinesia paradojal (KP). Si bien la KP es una cualidad del sistema motor per se (Ballanguer et al., 2006), su ocurrencia es más notable en algunos trastornos del movimiento, como la EP, involucrando directamente las relaciones del sujeto con su entorno en un comportamiento del que puede (o no) beneficiarse el individuo.

La KP en la EP fue descripta por el neurólogo francés Alexandre Achille Souques en 1921, observando que ciertos pacientes que caminaban con gran dificultad eran capaces, sin embargo, de andar y correr en determinadas circunstancias. La KP ha sido observada tanto en situaciones de emergencia como en situaciones cotidianas pero, en ambos contextos, la ocurrencia surge de la relación entre las potencialidades del sujeto y la propuesta estimuladora de su entorno (cf, Bacigalupe \& Pujol, 2014). Esta relación es de aprendizaje en tanto puede permitir la formación de estructuras y la reestructuración de esquemas que puedan utilizarse en experiencias posteriores. Una característica central de este aprendizaje es que no requiere esfuerzo consciente ni para su formación ni para su actualización en el tiempo.

Entiéndase por aprendizaje la adquisición de un cambio comportamental como resultado de la experiencia con el ambiente (Blaze \& Roth, 2013), como una capacidad biológica que permite a los organismos modificar su comportamiento para adaptarse a los cambios impredecibles del medio en que viven (Morgado Bernal, 2014). Claramente el aprendizaje pone en juego las relaciones entre in- 
dividuo y ambiente, donde las cualidades del ambiente, tales como la novedad (Justel \& Psyrdellis, 2014) pueden ejercer una influencia preponderante en los procesos de memoria subyacentes.

La KP puede comprenderse dentro del contexto del aprendizaje implícito, entendido como el proceso mediante el cual los sujetos se vuelven sensibles a ciertas regularidades de su ambiente en ausencia de la intención de aprender y de conciencia de que uno está aprendiendo, de tal modo que el conocimiento resultante es difícil de expresar (Cleeremens, 1995, 2003; Shanks, 2005).

Los dos términos del concepto de aprendizaje implícito pueden ser justificados: por un lado, la KP muestra un componente central implícito. Esto aparece en distintos trabajos analizados, por ejemplo el estudio de Mazzoni, Hristova y Krakauer (2007) destaca la naturaleza implícita de la toma de decisiones que dependen del circuito dopaminérgico. Por otro lado, la KP incluye respuestas aprendidas, por ejemplo en el estudio de Keefe, Salomone, Zigmond y Stricker (1989), todos los animales que habían sido entrenados a pesar de su akinesia pudieron escapar rápidamente del baño saltando afuera del acuario, lo cual permitió analizar que el fenómeno podía incluir respuestas aprendidas y no sólo acciones motoras reflejas.

\section{MODELO DE LAS RELACIONES ENTRE PERCEPCIÓN Y MOVIMIENTO}

El fenómeno de la KP incluye, entonces, dos factores clave en relación mutua: (a) factores del entorno del individuo que actúan a través de su percepción y (b) factores del sistema motor del individuo que dan lugar al movimiento corporal.

Estos dos factores clave de la KP dependen de los sistemas perceptuales y motores, respectivamente, sistemas que están vinculados a nivel conductual y evolutivo y han evolucionado para sostenerse mutuamente (cf., Gazzaniga, Ivry \& Mangun, 2009).

La observación experimental de que la comprensión de la acción de los otros se co- rrelaciona con la activación de las mismas estructuras neurales que intervienen cuando uno mismo produce las acciones ha permitido el desarrollo del marco teórico de neuronas espejo (cf., por ejemplo Gallese, 2009; Gazzaniga et al., 2009), una de cuyas derivaciones es la provisión de una representación común para la percepción y la acción (Iacoboni, 2009), aun cuando la activación de las vías motoras podría ser subliminal, sin implicar movimiento observable (Borroni, Gorini, Riva, Bouchard \& Cerri, 2011).

El concepto de acoplamiento (coupling) supone la existencia de una relación causal circular continua entre la percepción y la acción (Montagne, Cornus, Glize, Quaine \& Laurent, 2000). Montagne y sus colaboradores (2000) señalan que este acoplamiento sólo es posible si el sujeto está continuamente informado del estado del sistema actor-ambiente, donde el flujo visual puede jugar un rol (Ballanguer et al., 2006) aunque no es la única fuente de información relevante (Montagne, Bastin \& Jacobs, 2008). En contextos sociales puede sugerirse que en este acoplamiento hay una participación de la empatía como forma de cognición social (Moya-Albiol, Herrero \& Bernal, 2010).

Milner y Goodale (1995, 2006, ver Schenk $\&$ McIntosh, 2010) han propuesto un modelo de percepción-acción según el cual la visión existe en función de mejorar nuestras acciones. Existen dos vías en este acoplamiento: (a) la vía directa o corriente dorsal (dorsal stream) y (b) la vía indirecta o corriente ventral (ventral stream). Mientras que la vía dorsal se ocupa de la guía de la acción en tiempo real, el rol de la vía ventral en la acción se refiere al reconocimiento de patrones visuales (coincidiendo con la visión más clásica del término percepción). Ambas corrientes analizan la información visuoespacial de modo diferente de acuerdo con sus objetivos comportamentales. Schenk y McIntosh (2010) y Montagne y sus colaboradores (2008) discuten la distinción absoluta de los roles de ambas corrientes, proponiendo una interacción fluida que implica compartir algunas funciones y componentes.

En esta revisión de las interacciones de funciones perceptivas y motoras, Montagne y 
sus colaboradores (2008) sugieren redefinir el concepto de anticipación visual. Los autores ponen como ejemplo el juego deportivo, señalando que la información perceptual sobre la que confían los jugadores con frecuencia no es predictiva. De este modo, consideran que la anticipación visual se refiere a la habilidad de usar la información prospectiva en escala de unidades de máxima capacidad de acción. En este contexto, la experticia depende de la habilidad de establecer un acoplamiento entre el movimiento y las fuentes de información prospectiva.

Según estos autores, las cualidades de un objeto en relación a la acción (affordances) se presentan en escala de la acción: la percepción de estas cualidades (por ejemplo, la capturabilidad de una pelota en vuelo) es más acertada si el sujeto está en movimiento. Estas affordances constituyen recursos del medioambiente que proveen al individuo oportunidades de comportarse mediante su percepción. Esto significa que es la relación entre $o b$ jeto ambiental y percepción del individuo lo que constituye la affordance (Chamero, 2003): una affordance representa una relación entre el sistema de acción-percepción individual con las cualidades ambientales o contextos de aprendizaje. La novedad per se en un ambiente puede constituir una affordance, pues se ha observado que un ambiente novedoso contiene elementos que influyen sobre los procesos neurobiológicos de los sistemas de memoria que sostienen el aprendizaje (Justel \& Psyrdellis, 2014).

De acuerdo a la propuesta de Montagne y colaboradores (2008), el uso de la información en escala de unidades de acción permite al actor resolver dos problemas centrales al mismo tiempo: determinar el modo de acción (el qué -what- o vía ventral) y regular la acción (el cómo -how- o la vía dorsal). Consecuentemente, estos autores aseveran que el comportamiento de los jugadores in situ no puede ser entendido sin considerar las contribuciones interactivas de ambas corrientes: las funciones perceptivas ocurren en íntima relación con la propuesta medioambiental de acción.

De este modo, actuando a través de la KP el ambiente puede influir como contexto de aprendizaje sobre el accionar del individuo con EP. Este fenómeno puede estar influido por distintas variables personales que incluyen desde la motivación, como ya se ha dicho ( $\sin$ descartar la influencia del estilo de afrontamiento, cf., Santesmases-Montalbán, Donate-Martínez, Macías-Macías, Ordóñez-Camacho \& García-Moreno, 2010), el tipo de procesamiento más perceptual o más semántico (frecuentemente las tareas de memoria implícita se sostienen en el procesamiento perceptual, en contraste con tareas que implican un procesamiento más semántico o conceptual, cf,. Brunfaut \& d'Ydewalle, 1996), hasta la reserva cognitiva (cf., Poletti, Emre \& Bonuccelli, 2011; en cuanto a su conceptualización Allen, Bruss \& Damasio, 2005; Brayne et al., 2010; Stern, 2002, 2006; Stern et al., 2005; Tucker-Drob, Johnson \& Jones, 2009).

\section{Objetivos}

Teniendo en cuenta lo antedicho y considerando la existencia de la KP como característica del sistema motor, se propuso que en la EP están sensibilizadas las relaciones percepción-movimiento y que estas pueden ser modificables mediante la intervención oportuna en el ambiente del sujeto.

Dada la importancia de la percepción y del rol de las claves externas en el aprendizaje del movimiento, la intención fue averiguar si las cualidades de acción o affordances de ciertos objetos podían alterar la ejecución motora.

El objetivo fue indagar, en primer lugar, diferencias en la ejecución entre los participantes según la presencia de EP. Se esperaba en este caso que las personas con EP presentaran un tiempo de reacción mayor que los controles sin EP.

En segundo lugar, se analizó si había diferencias en el tiempo de reacción entre situaciones donde el estímulo sensorial era compatible (es decir, tanto la imagen presentada como la flecha superpuesta a la misma se orientaban hacia la misma dirección - ver Instrumentos y Procedimiento) y no compatible, y si estas diferencias se asociaban a ambos grupos o solamente a personas con EP. 
En este segundo caso se supuso que las personas con EP, al presentar la propiedad de la kinesia paradojal, podrían manifestar una mayor sensibilidad a las affordances del ambiente y por ende su ejecución podría estar más influenciada por la percepción del estímulo.

Finalmente, con esos resultados se esperaba agregar evidencia empírica a la propuesta teórica de un sistema integrado o de acoplamiento entre percepción y acción.

\section{MÉTOdOS}

PARTICIPANTES

Participaron del estudio 14 personas, que conformaron dos grupos: el grupo con Enfermedad de Parkinson (grupo EP) integrado por 9 personas y 5 integraron el grupo sin Enfermedad de Parkinson (grupo no-EP). Posteriormente se excluyó un participante del grupo EP por no cumplir con los criterios mínimos de inclusión, quedando el grupo constituido por 8 personas con EP.

Todos los participantes debían cumplir con los siguientes criterios de inclusión: tener como mínimo 55 años de edad, vivir en el Partido de La Plata o en el Gran Buenos Aires y presentar un desempeño cognitivo normal, medido con el Mini-Mental State Examination (MMSE - Grupo de Trabajo de Neuropsicología Clínica SNA, 1999).

Ambos grupos se formaron emparejando la edad y procedencia socioeconómica, dado que en la mayoría de los casos los sujetos del grupo control era la pareja o un hermano del enfermo con Parkinson.

Todos los integrantes del grupo EP tenían Enfermedad de Parkinson ideopática con severidad media (Hoehn \& Yahr: M: 2.12, $D E$ : .74; 4 hombres, 4 mujeres; rango: 1-3.5) (cf., Bermejo Pareja, Porta-Etessam, Díaz Guzmán \& Martínez-Martín, 2008). Todos los pacientes fueron evaluados en período on, es decir, en el período de movilidad mejorada durante el cual el paciente responde a la medicación antiparkinsoniana (en general, levodopa o L-DOPA, que se comercializa combinada con carbidopa o con benserazida). Un participante no estaba medicado con levodopa al momento de la ejecución de la prueba; cabe aclarar que fue incluido en el esquema de medicación en la consulta inmediata posterior con su neurólogo. En la Tabla 1 se informan la dosis diaria de levodopa de cada participante con EP y el valor obtenido en la prueba MMSE.

Los participantes firmaron un consentimiento informado de acuerdo a la Declaración de Helsinki de la Asociación Médica Mundial, Finlandia (1964) y sus enmiendas.

\section{INSTRUMENTOS Y PROCEDIMIENTO}

Se desarrolló una aplicación informática en colaboración con especialistas en Ingeniería de Sistemas en base a Oguro, Ward, Bracewel, Hindle y Rafal (2009).

En el estudio controlado reportado por estos autores, los estímulos aparecían en un fondo blanco de un monitor de video, ubicado 57 $\mathrm{cm}$. al frente de los participantes en posición sentada (17 personas con Enfermedad de Parkinson idiopática y 13 controles equiparados en edad, sin demencia ni otras condiciones neurológicas), quienes respondían con el teclado de una computadora Macintosh Powerbook G3, usando el software Psycope versión 1.2.5 para presentar los estímulos, medir el tiempo de reacción y las tasas de error.

Después de que los participantes iniciaban cada ensayo presionando la barra espaciadora, aparecía una imagen centralizada de un objeto facilitador o prime object: una imagen coloreada de una sartén con el asa dirigida hacia el observador y orientada, con igual probabilidad, hacia la mano izquierda o derecha del participante. La orientación y profundidad del asa simulaba una affordance para asir con la mano derecha o la mano izquierda.

Después de 1.200 milisegundos se proyectaba en el centro de la sartén una flecha pequeña que apuntaba hacia la derecha o la izquierda y se instruía al participante para que presionara cualquier tecla del lado izquierdo del teclado con la mano izquierda o cualquier tecla del lado derecho con la mano derecha. La relación entre el target y la orientación del asa 
de la sartén era al azar de manera tal que la affordance activada por el facilitador fuera igualmente probable de ser compatible (coincidir con la dirección del target) o incompatible (ser opuesto a la dirección del target). Los autores aplicaron series de 200 ensayos una vez que los sujetos participantes se habían habituado a la tarea.

En el estudio que se informa se realizó una aplicación similar en algunos aspectos pero diferente en otros. La aplicación fue instalada en una Notebook (Compaq Presario V3115LA), y en ella se trabajó con imágenes predeterminadas a fin de llevar a cabo el experimento. Los participantes se ubicaban sentados frente a la pantalla, a una distancia confortable para cada uno, donde se desplegaba en un fondo neutro la imagen facilitadora. Si bien se consideraron distintas imágenes, la única imagen facilitadora utilizada en el experimento final fue la de un sartén (ver Figura 1). Al cabo de distintos tiempos se proyectaba la flecha target por debajo del asa de la imagen. El número de ensayos en el presente estudio fue diferente al aplicado en el estudio de Oguro y colaboradores (2009).

La prueba se llevó a cabo en el hospital público, consultorio médico u hogar de los participantes, favoreciendo la familiaridad del participante con el contexto de trabajo, suponiendo a priori que la falta de familiaridad podía constituir un elemento distractor o estresor en contra de la mejor ejecución del participante. Diversos autores han trabajado en el beneficio que la familiaridad con el contexto del test puede ejercer sobre la ejecución, sea en animales no humanos o humanos (cf., por ejemplo Brown, 2001; De Rosa \& Patalano, 1991; Macken, 2002).

El procedimiento consistió en presentar en la pantalla la imagen de una sartén e instruir a los sujetos para que fijaran la mirada en la pantalla $y$, sin prestar atención a la imagen, atendieran a la flecha que aparecería superpuesta a la misma unos milisegundos después, pidiéndoles que presionaran una tecla cualquiera del lado del teclado al que apuntaba la flecha. Las imágenes consistían en fotografías color sobre un fondo neutro. Como se señaló anteriormente, si bien la aplicación informática incluía cuatro imágenes seleccionadas por sus cualidades de acción (cuchara, jarra, martillo y sartén), se escogió una de ellas para trabajar en el estudio (sartén) a fin de simplificar la cantidad de variables.

Los tiempos de presentación de la flecha (a partir de la aparición de la imagen) fueron 300, 900 y $1200 \mathrm{mseg}$. para ambos grupos.

Dos situaciones eran posibles en las relaciones flecha-imagen (ver Figura 1): compatibilidad (flecha apuntando a la misma dirección que la cualidad de acción de la imagen, representada por el mango del martillo y el asa de la sartén) o incompatibilidad (direcciones contrarias entre flecha y mango o asa). Cabe aclarar que la compatibilidad o incompatibilidad estaba programada para que ocurriera de forma azarosa, de manera que los participantes no pudieran prevenir la dirección de la flecha a aparecer.

Asimismo, dos eran las posibles respuestas del participante: acierto (presionar una tecla del lado del teclado al que apuntaba la flecha) y no-acierto (presionar una tecla del lado opuesto del teclado).

Previamente a la aplicación de la prueba los participantes se habituaron a la herramienta repitiendo la tarea la cantidad de ensayos necesaria hasta aprender el procedimiento. Se aplicaron 50 ensayos por participante, cantidad que equilibraba repetición necesaria con rapport.

\section{VARIABLES Y ANÁLISIS DE DATOS}

Las variables consideradas fueron compatibilidad y existencia de la Enfermedad de Parkinson como factores incidentes en el tiempo de reacción durante el experimento. La variable tiempo de reacción fue transformada a recíproca para normalizar la estructura de los residuos.

Además, dada la existencia de experimentos repetidos sobre los pacientes, se consideró oportuno incluir el número de ensayos como variable independiente, cuya hipotética incidencia indicaría una variación en el tiempo de respuesta en el transcurso de los ensayos, fuera ésta positiva o negativa. 
Cabe aclarar que a fin de no multiplicar las variables en este primer estudio, se tomaron los tiempos de presentación de la flecha como bloque, sin discriminar entre ellos a la hora del análisis. El nivel de significación elegido fue Alpha igual a .05.

Así como ocurrió con algunas características del procedimiento, instrumento y muestra, el análisis de datos fue distinto al aplicado por Oguro y colaboradores (2009), quienes utilizaron ANOVA para probar las hipótesis de tiempos de reacción y compatibilidad-incompatibilidad taget-objeto facilitador y Rho de Spearman para correlacionar la ejecución con la afectación motora en pacientes.

\section{REsultados}

La variable tiempo de reacción fue transformada en su inversa para linealizar su comportamiento como función del resto de las variables consideradas.

Se utilizó un modelo lineal general (MLG) para explorar el grado de relación entre las variables (McCullagh \& Nelder, 1989). Adicionalmente, para modelar adecuadamente el grado de incidencia en el tiempo de reacción producido por sucesivos ensayos realizados sobre cada individuo, se utilizó un modelo generalizado lineal mixto (MLGM) para mediciones repetidas (Faraway, 2006).

Se consideraron como efectos fijos a las variables presencia de Enfermedad de Parkinson, edad y compatibilidad, mientras que como efecto aleatorio se incluyó el número de ensayo. Para los cálculos se utilizó el software estadístico $R$ (R Development Core Team, 2011), con la rutina "Ime4".

La muestra total final quedó constituida por 1.050 ensayos.

El ajuste del MLG a la variable inversa de tiempo de reacción indica un aumento del tiempo de reacción con la edad y con la presencia de la Enfermedad de Parkinson, registrándose valores significativos de $p$ para ambas variables (edad y EP) dentro del modelo (ver Tabla 2).

De acuerdo a este resultado, la repetición de los ensayos tiene una alta incidencia, dis- minuyendo el tiempo de reacción, aún en individuos con EP. En la Figura 2 se observa una disminución del tiempo de reacción a lo largo de los ensayos para ambos grupos. Si bien el grupo con EP presenta un tiempo de reacción mayor, el patrón de aprendizaje no difiere con respecto a los controles (grupos sin $\mathrm{EP}$ ).

Considerando en segundo lugar la compatibilidad, se encontró que la misma incide en el tiempo de reacción solamente en las personas con EP (ver Tabla 3). En las personas sin EP la compatibilidad no incide significativamente en su tiempo de reacción.

\section{Discusión}

Los resultados obtenidos muestran que hubo un aumento del tiempo de reacción con la presencia de la Enfermedad de Parkinson (EP) y que, a medida que se sucedían los ensayos, el tiempo de reacción era menor.

Esto coincide con la bibliografía que indica tiempo de reacción aumentado en la ejecución de las personas con EP comparadas con sujetos sanos (cf. por ejemplo, Berry, Nicolson, Foster, Behrmann \& Sagar, 1999; Duru et al., 2012; Low, Miller \& Vierck, 2002; Paunikar, Shastri \& Baig, 2012). Asimismo el resultado coincide con la literatura sobre una mayor lentitud con el aumento de la edad (cf. por ejemplo, Sparrow, Begg \& Parker, 2006; Thompson, Blair \& Henrey, 2014; Tun \& Lachman, 2008).

La disminución del tiempo de reacción a medida que transcurrían los ensayos señala un aprendizaje de la tarea que no sucedió solamente en los controles sino también en las personas con EP, indicando que el aprendizaje puede estar intacto en estos sujetos. Este resultado coincide con la bibliografía que reporta aprendizaje durante la tarea en personas con EP (cf. por ejemplo, Press, Mechanic, Tarsy \& Manoach, 2002).

Sumado a lo dicho, se halló que la compatibilidad entre la imagen (sartén - dirección del asa) y el estímulo (flecha - dirección a la que apuntaba) influyó en el tiempo de reacción de las personas con EP pero no en los controles, lo cual podría indicar un efecto 
aumentado de la percepción sobre el movimiento en personas con Parkinson.

De este modo, en la muestra de personas de este estudio el tener EP influyó en el aumento del tiempo de reacción (en relación a los controles). Asimismo, es de suponer que tener EP puede haber incidido sobre la mayor sensibilidad perceptiva, de manera que la influencia de la compatibilidad imagen-estímulo sobre el tiempo de reacción podría estar dependiendo, al menos en parte, de tener o no la enfermedad.

Oguro y colaboradores (2009) llaman efecto de compatibilidad a la diferencia en tiempo de reacción entre situaciones compatibles y situaciones incompatibles, señalando que esta diferencia provee una medida de la fortaleza de la facilitación motora por las cualidades sensorio-motoras o affordances del objetoimagen. En su estudio, estos autores hallaron que las affordances facilitaban la respuesta motora (medida en tiempo de reacción) en personas con Enfermedad de Parkinson del mismo modo que en controles sanos.

Si bien en el estudio realizado se halló un efecto de compatibilidad, los resultados muestran que la compatibilidad influyó en la ejecución de las personas con Parkinson pero no en el grupo control. La diferencia con Oguro y colaboradores (2009) podría explicarse por las particularidades metodológicas que distinguen un estudio de otro y ya mencionadas previamente.

Que las personas con Parkinson hayan mostrado un efecto de compatibilidad se puede relacionar con la influencia que tiene la percepción sensorial en la facilitación motora. La teoría del control interno-externo (cf., Gazzaniga et al., 2009; Goldberg, 1985) señala que las personas con EP presentan un control interno de la programación motora deficitario (a cargo de la corteza motora suplementaria), de manera que para llevar adelante los programas motores voluntarios se requiere mayor preponderancia del control externo (mediante el accionar de la corteza premotora y sus relaciones con la áreas perceptuales del cerebro). Esto podría explicar la mayor sensibilidad de las personas con Parkinson a la estimulación sensorial y, por ende, la mayor influencia que la percepción de las cualidades de acción de los estímulos del ambiente puede tener sobre su acción.

Así los resultados obtenidos, agregan evidencia empírica a las perspectivas del acoplamiento entre percepción y acción (cf., por ejemplo, Montagne et al., 2000, 2008), apoyando la idea de que la percepción está al servicio de la acción y que existe una relación bidireccional entre ambos procesos constituyendo, posiblemente, un sólo sistema perceptivo-motor.

Esto señala un camino interesante para continuar indagando sobre las posibilidades que el diseño de contextos experienciales sensorio-estimuladores pueda aportar al mejoramiento de la movilidad de las personas con Enfermedad de Parkinson.

La idea de pensar la percepción sensorial en función de la acción (cf., por ejemplo, Montagne et al., 2000, 2008; Schenk \& McIntosh, 2010) constituye una cuestión fundamental para incluir en el análisis de las relaciones sujeto-entorno al trabajar en el aprendizaje del movimiento en personas con EP y en el aprendizaje humano en general.

El aprendizaje evaluado tiene la característica de ser implícito y no-consciente (cf., Cleeremens, 1995, 2003; Shanks, 2005). Como se ha reportado previamente, la kinesia paradojal es implícita (Exner, Koschack \& Irle, 2002; Mazzoni et al., 2007), implica aprendizaje (cf. Keefe, Salomone, Zigmond \& Stricker, 1989) y en su ocurrencia puede estar subyaciendo una reserva funcional (cf. Morris, Iansek, Matyas \& Summers, 1994) que se combina con cualidades del ambiente o claves contextuales (cf. por ejemplo, Akamatsu, Fukuyama \& Kawamata, 2008; Anzak et al., 2011; Fernández del Olmo \& Cudeiro, 2003; Fernández del Olmo, Arias, Furio, Pozo \& Cudeiro, 2006; Morris et al., 1994) para generar un movimiento de características sanas aún en personas con trastornos del movimiento como las personas con EP.

Cabe destacar las implicancias que estos resultados tienen para el estudio de los contextos de aprendizaje $(\mathrm{H}$. Lahitte, comunicación personal, marzo 2012). Si los resultados obtenidos son correctos, un contexto estimu- 
lador colabora potenciando la plasticidad de los sujetos para lograr un rendimiento mejorado en una tarea que pone a prueba las relaciones sensorio-motoras en el comportamiento humano. Un entorno de este tipo es un contexto de aprendizaje de resolución de problemas que promueve un deutero-aprendizaje (cf., Bateson, 1998) lo cual permite la transferencia a otros contextos. Estaríamos, entonces, ante una posibilidad de transferencia del aprendizaje o meta-plasticidad (cf., Herholz \& Zatorre, 2012), meta importante que el programa de indagación de los autores de este trabajo debe seguir interrogando.

Finalmente, los resultados informados tienen una generalización limitada y es necesario repetir el estudio en poblaciones con características diferentes para aumentar la validez de las conclusiones. Asimismo, en un estudio posterior sería interesante agregar la variable tiempo de presentación de la flecha al análisis asociada a la condición de tener o no Enfermedad de Parkinson, la compatibilidad imagen-estímulo y el tiempo de reacción.

TABLA 1

Valores del Mini Mental State Examination (MMSE) y dosis diaria de LEVOdOPA-CARBidopa o LEVOdOPA-BENSERAZIDA DE LOS PARTICIPANTES CON ENFERMEDAD DE PARKINSON

\begin{tabular}{|c|c|c|}
\hline Participante & MMSE $^{*}$ & L-DOPA $^{* *}$ \\
\hline 1 & 29 & 1000 \\
2 & 30 & 750 \\
3 & 30 & 1000 \\
4 & 30 & $0^{* * *}$ \\
5 & 27 & 1750 \\
6 & 28 & 437.5 \\
7 & s/d & 500 \\
8 & 29 & 750 \\
\hline
\end{tabular}

*Valor de corte 26 puntos

**Dosis diaria de levodopa-carbidopa o levodopa-benserazida (en mg.)

***Paciente que aún no se le ha indicado levodopa 
TABLA 2

InCIDENCIA DE LA EDAd Y DE LA PRESENCIA DE ENFERMEdAd DE PARKINSON (EP) SOBRE EL TIEMPO DE REACCIÓN (MLG)

\begin{tabular}{|l|c|c|c|c|}
\hline & Estimación & $\begin{array}{c}\text { Error } \\
\text { estándar }\end{array}$ & $t$ & $p$ \\
\hline Constante & 3.70 & .267 & 13852 & $<2 \mathrm{e}-16$ \\
Edad & -.03 & .004 & -9491 & $<2 \mathrm{e}-16$ \\
EP & -.58 & .050 & -11797 & $<2 \mathrm{e}-16$ \\
\hline
\end{tabular}

TABLA 3

INCIDENCIA DE LA COMPATIBILIDAD SOBRE EL TIEMPO DE REACCIÓN (ENSAYO) EN EL GRUPO CON ENFERMEDAD DE PARKINSON (EP)

\begin{tabular}{|l|c|c|c|c|}
\hline & Estimación & $\begin{array}{c}\text { Error } \\
\text { estándar }\end{array}$ & $t$ & $p$ \\
\hline Constante & .001312 & .0001771 & 7.40 & $<.0001$ \\
Edad & .000521 & .0002243 & 2.32 & .0103 \\
EP & .000004 & .000001325 & 2.99 & .0014 \\
Compatibilidad: EP & .000153 & .00002842 & 5.37 & $<.0001$ \\
\hline
\end{tabular}


FIGURA 1

IZQUIERDA: SITUACIÓN COMPATIBLE FLECHA - IMAGEN; DERECHA: SITUACIÓN INCOMPATIBLE FLECHA - IMAGEN (EP)

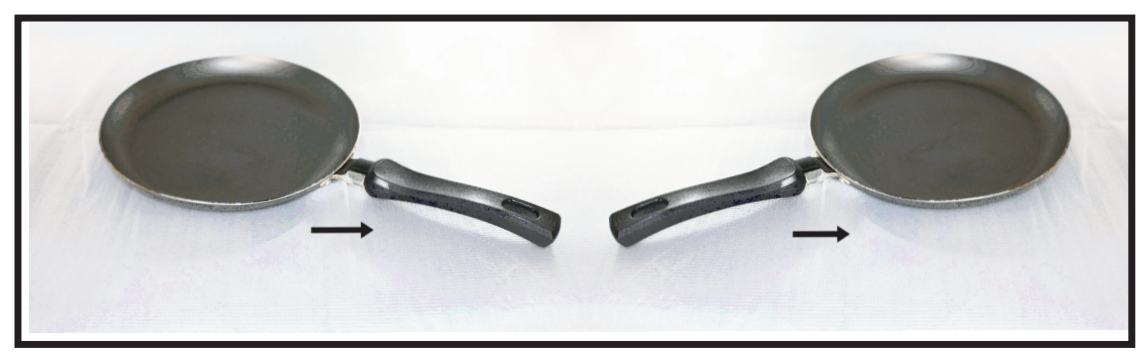

FIGURA 2

INCIDENCIA DEL NÚMERO DE ENSAYOS SOBRE EL TIEMPO DE REACCIÓN DE LOS INDIVIDUOS

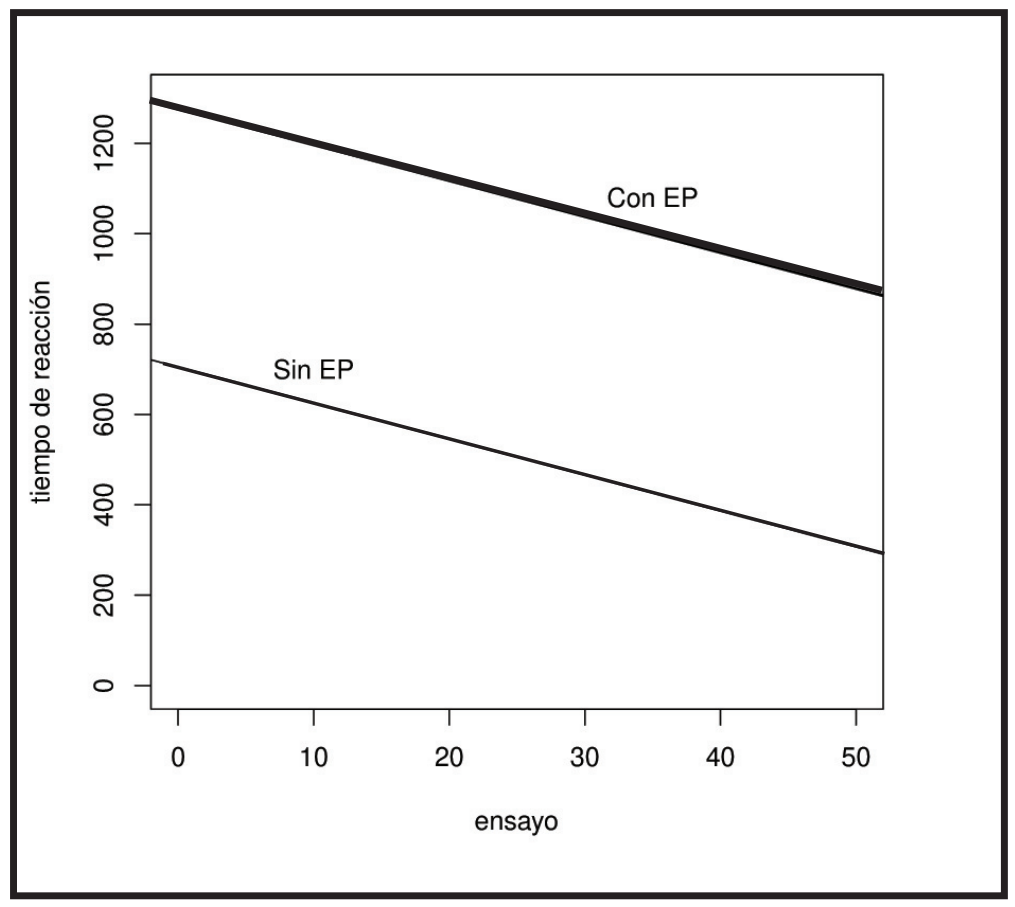

Nota:

Participantes con EP: línea gruesa

Sin EP: línea fina

Ensayos $=$ Estimación $=-7.957$ 


\section{REFERENCIAS BIBLIOGRÁFICAS}

Akamatsu, T., Fukuyama, H. \& Kawamata, T. (2008). The effects of visual, auditory, and mixed cues on choice reaction in Parkinson's disease. Journal of the Neurological Sciences, 269, 118-125. http://dx.doi.org/10.1016/j.jns.2008.0 1.002

Allen, J.S., Bruss, J. \& Damasio, H. (2005). The aging brain: The cognitive reserve hypothesis and hominid evolution. American Journal of Human Biology, 17, 673-689. http://dx.doi.org/ 10.1002/ajhb.20439

Anzak, A., Tan, H., Pogosyan, A., Djamshidian, A., Ling, H., Lees, A. et al. (2011). Improvements in rate of development and magnitude of force with intense auditory stimuli in patients with Parkinson's disease. European Journal of Neuroscience, 34(1), 124-132. http://dx.doi.org/10. 1111/j.1460-9568.2011.07735.x

Bacigalupe, M.A. \& Pujol, S. (2014). El movimiento desde la perspectiva de la neurociencia social cognitiva: El caso de la Enfermedad de Parkinson [Movement from the social cognitive neuroscience: The case of Parkinson's disease]. Vertex, 25(118), 429-436.

Ballanguer, B., Thobois, S., Baraduc, P., Turner, R. S., Broussolle, E. \& Desmurget, M. (2006). "Paradoxical kinesis" is not a hallmark of Parkinson's disease but a general property of the motor system. Movement Disorders, 21(9), 1490-1495.

Bateson, G. (1998). Pasos hacia una ecología de la mente [Steps to an ecology of mind]. Buenos Aires: Lohlé-Lumen.

Bauso, D.J., Tartari, J.P., Stefani, C.V., Rojas, J.I., Giunta, D.H. \& Cristiano, E. (2012). Incidence and prevalence of Parkinson's disease in Buenos Aires City, Argentina. European Journal of Neurology, 19(8), 1108-1113. http://dx.doi.or g/ 10.1111/j.1468-1331.2012.03683.x

Bermejo Pareja, F., Porta-Etessam, J., Díaz Guzmán, J. \& Martínez-Martín, P. (2008). Más de cien escalas en neurología [More than a hundred scales in neurology]. España: Aula Médica Ediciones. Recuperado el 3 de marzo de 2009 de www.neuroloxia.com/wp.../2009//esca las en_neurologia_marzo.pdf

Berry, E.L., Nicolson, R.I., Foster, J.K., Behrmann, M. \& Sagar, H.J. (1999). Slowing of reaction time in Parkinson's disease: the involvement of the frontal lobes. Neuropsychologia, 37(7), 78795. http://dx.doi.org/10.1016/s0028-3 932(98) 00137-7

Blaze, J. \& Roth, T.L. (2013). Epigenetic mechanisms in learning and memory. Wiley Interdisciplinary Reviews: Cognitive Science, 4(1), 105-115. https://doi.org/10.1002/wcs.1205

Borroni, P., Gorini, A., Riva, G., Bouchard, S. \& Cerri, G. (2011). Mirroring avatars: Dissociation of action and intention in human motor resonance. European Journal of Neuroscience, 34(4), 662-669. http://dx.doi.org/10.1111/j.146 0-9568.2011.07779.x

Brayne, C., Ince, P., Keage, H., McKeith, I., Matthews, I., Polvikoski, T. et al. (2010). Education, the brain and dementia: neuroprotection or compensation? Brain, 133, 2210-2216. http: //dx.doi.org/10.1093/brain/awq185

Brown, C. (2001). Familiarity with the Test Environment Improves Escape Responses in the Crimson Spotted Rainbowfish, Melanotaenia duboulayi. Animal Cognition, 4(2), 109-113. https://doi.org/10.1007/s100710100105

Brunfaut, E. \& d'Ydewalle, G. (1996). A comparison of implicit memory tasks in Korsakoff and alcoholic patients. Neuropsychologia, 34(12), 1143-1150. http://dx.doi.org/10.1016/0028-393 2(96)00032-2

Chamero, A. (2003). An outline of a theory of affordances. Ecological Psychology, 15(2), 18195. http://dx.doi.org/10.1207/s15326969eco1 $502 \_5$

Cleeremans, A. (1995). Implicit learning in the presence of multiple cues. En Proceedings of the 17th Annual Conference of the Cognitive Science Society (pp. 1-6). Hillsdale, NJ: Erl- 
baum. Recuperado el 10 de febrero de 2011 de http://citeseerx.ist.psu.edu/viewdoc/download

Cleeremans, A. (2003). Models of implicit learning. En L. Nadel (Ed.), Encyclopedia of cognitive sciences (Vol. 2, pp. 491-499). London: Macmillan Publishers.

Crucian, G., Huang, L., Barrett, A., Schwartz, R., Cibula, J., Anderson, J. et al. (2001). Emotional conversations in Parkinson's disease. Neurology, 56, 159-165. http://dx.doi.org/10.1212/wn 1.56.2.159

De Rosa, A. \& Patalano, F. (1991). Effect of familiar proctor on fifth and sixth grade student's test anxiety. Psychological Report, 68, 103-113. https://doi.org/10.2466/pr0.1991.68.1.103

Duru, C., Lelard, T., Roussel, M., Yerro, Y., Godefroy, O. \& Krystkowiak, P. (2012). Mechanisms slowing in Parkinson's disease [Abstract]. Movement Disorders, 27(Suppl 1), 45.

Exner, C., Koschack, J. \& Irle, E. (2002). The differential role of premotor frontal cortex and basal ganglia in motor sequence learning: Evidence from focal basal ganglia lesions. Learning \& Memory, 9, 376-386. http://dx.doi.org/ $10.1101 / \mathrm{lm} .48402$

Faraway, J. (2006). Extending linear model with $R$. London: Chapman and Hall.

Fernández del Olmo, M. \& Cudeiro, J. (2003). A simple procedure using auditory stimuli to improve movement in Parkinson's disease: A pilot study. Neurology \& Clinical Neurophysiology, (2), 1-7. Recuperado el 10 de febrero de $2011 \mathrm{de}$ http://www.neurojournal.com/article/ viewFile/445/380

Fernández del Olmo, M., Arias, P., Furio, M., Pozo, M. \& Cudeiro, J. (2006). Evaluation of the effect of training using auditory stimulation on rhythmic movement in Parkinsonian patients a combined motor and [18F]-FDG PET study. Parkinsonism \& Related Disorders, 12, 155164. http://dx.doi.org/10.1016/j.parkreldis. 200 5.11 .002

Gallese, V. (2009). Motor abstraction: A neuroscientific account of how action goals and in- tentions are mapped and understood. Psychological Research, 73(4), 486-498. http://dx.doi. org/10.1007/s00426-009-0232-4

Gazzaniga, M., Ivry, R. \& Mangun, G. (2009). Cognitive neuroscience: The biology of the mind. New York: WW Norton \& Company.

Goldberg, G. (1985). Supplementary motor area structure and function: Review and hypotheses. Behavioral and Brain Sciences, 8, 567588. http://dx.doi.org/10.1017/s0140525x000 45167

Grupo de Trabajo de Neuropsicología Clínica de la Sociedad Neurológica Argentina. (1999). El "Mini-Mental State Examination" en la Argentina: Instrucciones para su administración [The "Mini-Mental State Examination" in Argentine: Instructions for administration]. Revista de Neurología Argentina, 24(1), 31-35.

Herholz, S. \& Zatorre, R. (2012). Musical training as a framework for brain plasticity: Behavior, function, and structure. Neuron, 76, 486-502. http://dx.doi.org/10.1016/j.neuron.2012.10.011

Holtgraves, T. \& Cadle, C. (2016). Communication impairment in patients with Parkinson's disease: Challenges and solutions. Journal of Parkinsonism and Restless Legs Syndrome, 6, 45-55. http://dx.doi.org/10.2147/jprls.s83164

Iacoboni, M. (2009). Imitation, empathy, and mirror neurons. Annual Review of Psychology, 60, 653-670. http://dx.doi.org/10.1146/annurev.ps ych.60.110707.163604

Justel, N. \& Psyrdellis, M. (2014). Novedad y modulación de la memoria: Mecanismos neurobiológicos implicados [Novelty and modulation of memory: Neurobiological mechanisms involved]. Interdisciplinaria, 31(2), 195-211. https: //doi.org/10.16888/interd.2014.31.2.1

Keefe, K., Salomone, J., Zigmond, M. \& Stricker, E. (1989). Paradoxical kinesia in parkinsonism is not caused by dopamine release. Archives of Neurology, 46, 1070-1075. http://dx.doi.org/1 0.1001/archneur.1989.00520460046012

Low, K.A., Miller, J. \& Vierck, E. (2002). Response slowing in Parkinson's disease: A psy- 
chophysiological analysis of premotor and motor processes. Brain, 125(Pt 9), 1980-1994. http://dx.doi.org/10.1093/brain/awf206

Macken, W. (2002). Environmental context and recognition: The role of recollection and familiarity. Journal of Experimental Psychology: Learning, Memory and Cognition, 28(1), 153161. http://doi.org/10.1037//0278-393.28.1.153

Mazzoni, P., Hristova, A. \& Krakauer, J. (2007). Why don't we move faster? Parkinson's disease, movement vigor, and implicit motivation. Journal of Neuroscience, 27(27), 7105-7116. http:// dx.doi.org/10.1523/jneurosci.0264-07.20 07

McCullagh, P. \& Nelder, J. (1989). Generalized linear models. London: Chapman and Hall.

Montagne, G., Bastin, J. \& Jacobs, D. (2008). What is visual anticipation and how much does it rely on the dorsal stream? International Journal of Sport Psychology, 39(2), 149-156.

Montagne, G., Cornus, S., Glize, D., Quaine, F. \& Laurent, M. (2000). A perception-action coupling type of control in long jumping. Journal of Motor Behavior, 32(1), 37-43. http://dx.doi.org/ $10.1080 / 00222890009601358$

Morgado Bernal, I. (2014). Naturaleza y origen del aprendizaje y la memoria [Nature and origin of human learning]. En I. Morgado Bernal (Ed.), Aprender, recordar y olvidar: Claves cerebrales de la enseñanza eficaz (pp. 23-39). España: Ariel.

Morris, M., Iansek, R., Matyas, T. \& Summers, J. (1994). Ability to modulate walking cadence remains intact in Parkinson's disease. Journal of Neurology, Neurosurgery and Psychiatry, 57, 1532-1534. http://dx.doi.org/10.1136/ jnnp.57. 12.1532

Moya-Albiol, L., Herrero, N. \& Bernal, C. (2010). Bases neuronales de la empatía [Neural basis of empathy]. Revista de Neurología, 50(2), 89100.

Oguro, H., Ward, R., Bracewel, M., Hindle, J. \& Rafal, R. (2009). Automatic activation of motor programs by object affordances in patients with Parkinson's disease. Neuroscience Letters, 463
(1-10), 35-36. http://dx.doi.org/10.1016/j.neu let.20 09.07.033

Paunikar, V., Shastri, N. \& Baig, M. (2012). Effect of Parkinson's disease on audiovisual reaction time in Indian population. International Journal of Biological and Medical Research, 3(1), 13921396. Recuperado el 20 de julio de 2016 de http://www.biomedscidirect.com/482/effe ct_of_parkinson_s_disease_on_audiovisual_ reaction_time_in_indian_population/articlescategories

Poletti, M., Emre, M. \& Bonuccelli, U. (2011). Mild cognitive impairment and cognitive reserve in Parkinson's disease. Parkinsonism \& Related Disorders, 17, 579-586. http://dx.doi. org/10.1016/j.parkreldis.2011.03.013

Press, D., Mechanic, D., Tarsy, D. \& Manoach, D. (2002). Cognitive slowing in Parkinson's disease resolves after practice. Journal of $\mathrm{Neu}$ rology, Neurosurgery and Psychiatry, 73, 524528. http://dx.doi.org/10.1136/jnnp.73.5.524

Pringsheim, T., Jette, N., Frolkis, A. \& Steeves, T. (2014). The prevalence of Parkinson's disease: A systematic review and meta-analysis. Movement Disorders, 29(13), 1583-1590. http://dx. doi.org/10.1002/mds.25945

R Development Core Team. (2011). $R$ : A language and environment for statistical computing. Vienna: R Foundation for Statistical Computing.

Santesmases-Montalbán, I., Donate-Martínez, S., Macías-Macías, I., Ordóñez-Camacho, X. \& García-Moreno, L. (2010). Funciones ejecutivas y estrategias de afrontamiento en la enfermedad de Parkinson idiopática [Executive functions and coping strategies in idiopatic Parkinson's disease]. Psicogeriatría, 2(4), 243-246.

Schenk, T. \& McIntosh, R. (2010). Do we have independent visual streams for perception and action? Cognitive Neuroscience, 1(1), 52-78. http://dx.doi.org/10.1080/1758892090338895 0

Shanks, D. (2005). Implicit learning. En K. Lamberts \& R. Goldstone (Eds.), Handbook of cognition (pp. 202-220). London: Sage. 
Souques, A. A. (1921). Rapport sur les syndromes parkinsoniens [Report on Parkinsonism syndromes]. Revue Neurologique, 37, 534-573.

Sparrow, W.A., Begg, R.K. \& Parker, S. (2006). Aging effects on visual reaction time in a single task condition and when treadmill walking. Motor Control, 10, 201-211. http://dx.doi.org/ 10.1123/mcj.10.3.201

Stern, Y. (2002). What is cognitive reserve? Theory and research application of the reserve concept. Journal of the International Neuropsychological Society, 8, 448-460. http://dx.doi.org/10. $1017 / \mathrm{s} 1355617702813248$

Stern, Y. (2006). Cognitive reserve and Alzheimer disease. Alzheimer Disease and Associate Disorders, 20(2), 112-117. http://dx.doi.org/10.10 97/01.wad.0000213815.20177.19

Stern, Y., Habeck, C., Moeller, J., Scarmeas, N., Anderson, K., Hilton, H. et al. (2005). Brain networks associated with cognitive reserve in healthy young and old adults. Cerebral Cortex,
15, 394-402. http://dx.doi.org/10.1093/cercor/ bhh142

Thompson, J.J., Blair, M.R. \& Henrey, A.J. (2014). Over the hill at 24: Persistent age-related cognitive - motor decline in reaction times in an ecologically valid video game task begins in early adulthood. Plos One, 9(4): e94215. http:// dx.doi.org/10.1371/journal.pone.0094215

Tucker-Drob, E., Johnson, K. \& Jones, R. (2009). The cognitive reserve hypothesis: A longitudinal examination of age-associated declines in reasoning and processing speed. Developmental Psychology, 45(2), 431-446. http://dx. doi.org/10.1037/a0014012

Tun, P.A. \& Lachman, M.E. (2008). Age differences in reaction time and attention in a national telephone sample of adults: Education, sex, and task complexity matter. Developmental Psychology, 44(5), 1421-1429. http://dx.doi.org/10.10 $37 / \mathrm{a} 0012845$ 